\title{
An Algorithm for Understanding Spine Imaging
}

\author{
Joseph Fortin, DO
}

The presentation of a patient with primary spine pathology may be confounded by a myriad of symptoms that span many organ systems. Likewise, imaging findings are often diverse and may be as subtle as slight posterior joint asymmetry combined with paravertebral myofascial strain or as gross as fracture-dislocation with neurological sequelae.

A systematic approach to selecting and applying the appropriate imaging study combined with a careful clinical history and examination will insure a more accurate diagnosis and proper treatment. The biomechanics of injury and the

The spine is intimately connected to a host of adjacent and remote neurovascular, osteoligamentous, myofascial and visceral structures. Hence, the presentation of a patient with primary spine pathology may be confounded by a myriad of symptoms that span many organ systems.

Likewise, imaging findings are often diverse and may be as subtle as slight posterior joint asymmetry combined with paravertebral myofascial strain or as gross as fracture-dislocation with neurological sequelas. This potentially complex imaging puzzle engenders the need for the systematic application of various imaging modalities in solving each case.

\section{PHYSICAL AND TECHNICAL PRINCIPLES}

An image of an object is a graphical representation of the spatial distribution of one or more of its properties. An image is a reflection of not only the structure being observed, but also of the physical principles of the imaging modality.

From Spine Technology and Rehabilitation, Fort Wayne, Indiana. Dr. Fortin is the medical director of Spine Technology and Rehabilitation and clinical professor of Indiana University School of Medicine. Address correspondence: Joseph Fortin, DO, 7320 Eagle Road, \#210, Fort Wayne, Indiana 46804. E-mail: fortin@ pol.net pathophysiology of the disease process in question should always be considered.

This review discussed an algorithmic approach for understanding spine imaging with discussions of radiography, radionuclide scan, myelography, computed tomography scanning and magnetic resonance imaging.

Keywords: Spine imaging, radiography, radionuclide scan, myelography, computed tomography scanning, magnetic resonanceimaging

\section{Radiography}

Despite orthogonal, multidimensional and other imaging technologies available, radiographs still fulfill a basic role within our diagnostic armamentarium for screening trauma victims, ruling out instability or segmental motion aberration, ascertaining segmentation, and providing exquisite bone detail. The spatial resolution of transmission radiography provides a unique perspective on bone morphology and texture that remains unsurpassed. Hence, imaging experts still commonly rely on the radiographic images to complement other modalities, in evaluating giant cell tumor, hemangionia, Paget's disease, osteoid osteoma, osteochondritis dessicans and other osseous findings.

Plain films or X-rays are often the screening modality of choice for patients presenting to the Emergency Room who have sustained traumatic injuries. For example, a cross table lateral projection allows the physician a "quick peek" at all four basic anatomical lines of $\mathrm{C}$-spine alignment, i.e. retropharyngeal, pre- and post vertebral body and interiaminar. Flexion/extension and lateral bending X-rays provide information on the dynamic integrity of the spinal column. Radiographs may disclose frank fracture-dislocation, retropharyngeal edema, tracheal displacement, or instability. However, several authors have demonstrated a high incidence of cervical spine pathology despite normal radiography $(1,2)$.

The process of acquiring plain films or radiographs involves positioning an X-ray tube opposite the interposed body 
part and film cassette. Within the X-ray tube, electrons are "boiled off" a heated cathode filament. Subsequently, the electrons are rapidly accelerated across high voltage, striking an anode target, which results in the production of electromagnetic energy (i.e., X-rays) and heat. The emerging beam is constricted by collimators to the area being imaged (3). X-rays which have not been absorbed or scattered (by structures in the patient) then strike the film cassette. Within the cassette, rare earth phosphorus atoms efficiently capture the $\mathrm{x}$-ray photons and emit visible light which exposes photographic film. A physiochemical reaction in the film ensues, according to the radiographic density and volume of the interposed body part, resulting in an image (4). The fundamental radiographic densities are water density (i.e. muscle), fat density, air density, and calcium density (bone).

An x-ray beam is diverging; therefore, objects closer to the $\mathrm{x}$-ray tube may be magnified. The relationships of the distances between the tube, patient structures, and film cassette affect the geometry of the image. The quality of plain radiographs depends upon tube, cassette, and patient positioning, exposure settings, film and cassette technology, as well as processing factors. Parenthetically, some large sites use filmless digital systems for radiography.

\section{Radionuclide Scan}

This imaging study involves scanning following the administration of a radioactive isotope, which is taken up by active bone. Gamma rays emitted by the tracer pass through a focusing collimator and interact with a crystal, creating scintillation. The resultant burst of light creates a voltage change in a photomultiplier tube, which feeds into a scintillation location circuit (that determines the position coordinates with the crystal of each scintillation event). The combined components (i.e. crystal, photomultiplier tube and scintillation location circuit) collectively are the ana$\log$ of retinal photoreceptors and associated neural network of an eye (15).

In 1964, a Computed Tomography (CT)-like radionuclide imaging device was invented, but only over the past decade has SPECT (single photon emission computed tomography) become the true counterpart of X-ray CT. The SPECT data can be displayed as either transaxial scans or three dimensional images. This presentation is possible by positioning one to four fairly standard gamma camera heads (each with collimator, crystal and PMT array) mounting them on a supporting gantry and rotating them around the patient following the administration of the radioactive tracer (3).

Bone scans are customarily used to determine if a fracture is physiologically active or as a screening modality for neoplasm or infection. Magnetic Resonance Imaging (MRI) has eclipsed radionuclide scanning for the evaluation of a putative discitis case. In general, radionuclide scanning is highly sensitive for bone infection and will disclose a "hot spot" several days before any abnormalities become visible on plain films.

Poor sensitivity and specificity have been recently documented when bone scans are applied to diagnose sacroiliac joint dysfunction and facet syndrome. Radionuclide scanning does not differentiate one physiologically active process from another. For instance, a patient with a "hot" zygapophyseal joint from degenerative arthritis may easily be misinterpreted as an active spondylolytic lesion.

Again, bone scans are etiologically nonspecific. A common example is a child who is limping and has a tender area on palpation with a positive radionuclide scan. The area of increased tracer uptake may be tumor or a healing stress fracture rather than an infection. Moreover, bone scans will remain positive for long periods of time after an infection has been eradicated and may be falsely negative with multiple myeloma and other lytic tumors (e.g., renal or thyroid).

\section{Myelography}

While doing a Myelography study, 5 to $15 \mathrm{~mL}$ of contrast medium is instilled in the subarachnoid space with a 25 gauge $3 \frac{1}{2}$ inch spinal needle, opacifying the subarachnoid space and outlining its neurovascular contents, e.g., dural root sleeve, conus and cord.

Clinical applications include: screening for tumor or CSF block, dynamic disc prolapse or root entrapment and combined with CT in the setting of stenosis. There are few indications for plain myelography since the advent of CT and MRI. CT and MRI provide three dimensional imaging and are noninvasive.

\section{Computed Tomography (CT) Scanning}

In the 1970s, CT provided the first direct views of discs, nerve roots, and spinal canals. Employing an array of electronic detectors directly opposite a rapidly rotating X-ray tube, CT produces cross-sectional images, opening the window for three-dimensional conceptualization of the 
spine.

The patient lies on a moveable bed placed within the imaging device or "gantry." Within the gantry, an X-ray tube rotates around the patient during the exposure (typically one second for a $360^{\circ}$ rotation), while an X-ray detector rotates on the side of the patient opposite the tube. A xenon or solid state detector captures the X-ray photons, which pass through the patient.

Tissues, which absorb or deflect a large number of X-ray photons are said to have high attenuation. In classic axial (non-helical) scanning, the pattern of attenuation detected during a single rotation (i.e., each exposure or slice) is computer-processed into a cross-sectional image. The thickness of that slice depends upon the collimation of the $\mathrm{X}$-ray beam. The number of slices needed depends upon the area to be covered, the thickness of each slice, and the relationship of adjacent slices, whether contiguous (most common), overlapped, or with some interslice gap $(3,4)$. Shades of gray for each point on the displayed image are chosen to represent the attenuation of the tissue at that point. Window and level controls on the CT computer allow for choices in gray scale (contrast) and brightness, which enhance the viewer's perception of various tissues. For example, a broad gray scale (wide window setting) provides greater bone detail, while a narrow window setting enhances soft tissue.

In classic axial CT scanning, each section viewed is the result of a single exposure as described above, followed by movement of the patient bed to the next slice location. In helical (also called "spiral") CT the entire anatomic area under study is scanned in a single long exposure while the tube and detector array make many rotations around the patient. Thus a cylindrical volume is scanned, and the data from that volume can be used to produce cross-sectional images. For mathematical reasons beyond the scope of this text, the effective width of each slice produced is slightly greater than the width-collimation-of the X-ray beam. Compared to axial scanning, some resolution is lost, especially along the axis of patient movement.

While the slice thickness of helical scanning is limited by the physical size of the $\mathrm{x}$-ray beam, the number of slices computed from the data acquisition has virtually no limitation beyond storage capacity and patience of the viewer. The scanner could produce, for example, slices with an effective width of $5.5 \mathrm{~mm}$, each slice beginning just $1 \mathrm{~mm}$ from the beginning of the previous slice, resulting in many greatly overlapped CT images. Most commonly, either contiguous sections or $50 \%$ overlapped sections are acquired (6).

Because a CT image represents a "slice" of tissue of a given thickness, each point on the image represents a volume, which could contain more than one tissue type. For example, a calcified pulmonary granuloma (very high attenuation) could "share" a location with aerated lung (very low attenuation). The measured attenuation for that point would be an average of the two tissues. The attenuation value of shade of gray at the position would thus represent neither tissue accurately. This artifact is known as "partial volume averaging." Understanding this limitation of CT is important for those who are responsible for determining CT protocols, as well as for all those who interpret CT images (7).

The measurement of attenuation by a tissue on a CT image is given in Houndsfield units (HU). Tissues measuring above 100 Houndsfield units usually contain calcium. Structures measuring near $0 \mathrm{HU}$ are water density, those near minus $100 \mathrm{HU}$ are fatty, and those near minus 1000 are air containing (3).

The images presented in axial scanning are usually just those cross-sections created in-plane with scanner, possibly oblique to the patient's anatomy, e.g., if they have scoliosis. In helical scanning, the volume acquisition allows more freedom to produce a variety of multiplanar displays, including curved planes following a deformity or parallel to a normal spinal curvature, as well as threedimensional shaded surface displays.

Despite the latest MR imaging techniques, newer pulse sequences, improved receiver coils, and computer postprocessing enhancement of MR images, CT still has higher spatial resolution for better definition of fractures and other bone morphology. CT provides excellent visualization of osseous detail, including post-traumatic changes, facet arthropathy, spondylolysis, spondylolisthesis, osseous stenosis, and structural alignment. Spatial and contour changes from disc pathology are adequately evaluated by $\mathrm{CT}$, yet beam attenuation may occur at the cervicothoracic and lumbosacral junctions. The main limitations of CT include radiation exposure, slightly restricted field of view, and poor delineation of intrathecal pathology. The overwhelming majority of soft tissue processes are best imaged by MRI; however, post-discography CT is more sensitive in detecting sometimes painful annular fissures, and post myelography CT may still be more sensitive than MRI in detecting nerve root avulsion (8). 
Routine posttraumatic cervical spine CT with multiplanar reconstructions should include contiguous thin transaxial sections form the craniocervical junction to the mid-pedicle of T1. High-resolution helical CT (collimation down to 1 $\mathrm{mm}$ ) may be required to define complex or subtle fractures and to prevent misregistration artifact. Overlapping slice reconstruction is necessary for high quality multiplanar reconstruction images. The speed of helical scanning usually overcomes the traditional difficulty of trying to get an acutely injured patient to hold still for imaging. The inferior margin of the foramen magnum must be included to exclude fracture of an occipital condyle.

Functional craniocervical CT involves scanning during axial rotation of the head in order to demonstrate dynamic CO-1 and C1-2 instability. CT data can be acquired in 2025 minutes with conventional CT but this takes only seconds with helical scanners.

Modern rapid scanning techniques significantly reduce radiation exposure yet the risk must be considered when ordering a CT examination. Plain film evaluations are, unfortunately, limited by their lack of sensitivity and specificity and changes noted on plain films (including posttraumatic deformation) may not manifest themselves until they are subacute or chronic.

\section{Magnetic Resonance Imaging}

In the 1980s MRI allowed us to view soft tissue with stunning resolution. For the first time, we could visualize changes in hydration in the nuclear matrix, tiny fissures in the annulus and detailed intrathecal anatomy.

MRI images are based on the interaction of nuclei (usually the single proton in hydrogen) within a magnetic field. A spinning nucleus will tend to align its axis with an external magnetic field. An applied radiofrequency (RF) pulse will force that spin out of alignment, causing the nucleus to "wobble" -the axis of spin will precess around the axis of the external field. When the radiofrequency pulse, which is detected by the receiver, coils. The frequency of this emitted RF pulse is related to the strength of the magnetic field.

Superimposed upon the main magnetic field are "gradient" fields, so that there is a slight rise in magnetic field strength from one end of the scanner to the other, as well as from side to side. The gradients, which vary the field strength, result in slight frequency variations in the RF signals emitted by protons in the intervals when the external or applied pulses are turned off. The frequency differences provide spatial information along two axes, the applied pulses are "phase-encoded" and the phase differences provide information for that third axis.

The very complicated radio signal emitted by the patient's protons (the radiofrequency "echoes") is detected by receiver coils, which are very sensitive antennae. This signal is computer-processed into a cross-sectional image using mathematical formulas similar to those used in CT scanning.

The MR signal of a given tissue is related to two independent time constants $\mathrm{T} 1$ (or longitudinal relaxation time) and T2 (or transverse relation time). These constants vary with the intrinsic physical properties of a given tissue and produce varied signal intensities or contrast within the images. T1 is the time required for the excited protons to return to their equilibrium state and T2 is the loss of coherence or harmonic convergence of the precessing nuclei following discontinuation of the radiofrequency signal. T1 "weighted" images emphasize the T1 properties of a tissue and are generated with a short TR (repetition time between radiofrequency signals) of 400-600 ms and a short TE or echo time of 15-30 ms. Fat, subacute, and chronic hemorrhage, as well as proteinaceous fluid all yield high signal on T1. T1 weighted images are known for their excellent delineation of anatomic structures and are sometimes thought of as "fat images." T2 weighted images reflect the state of hydration of the tissue and are produced with a long TR of 1500-3000 ms and long TE of 60-120 ms. Tissues which are rich in free or extracellular water will generate high signal on T2 (e.g., cerebrospinal fluid, necrotic or inflammatory tissue, cystic structures, nucleus pulposus, and tumors). Proton density or spin density images have long TR-like T2 weighted images, and short TElike T1 weighted images. Hence, they retain a significant amount of anatomic detail (like T1) yet structures which are rich in water yield high signal (like T2). Proton density images reflect the absolute number of mobile hydrogen protons in a tissue. Mineral-rich tissues such as cortical bone contain few mobile protons and generate very little signal in all pulse sequences. Gas, which contains no mobile hydrogen protons, generates no MRI signal. T2 weighted images are prone to degradation from motion artifact because of the long acquisition time. The above discussion refers to "conventional spin echo" sequence of RF pulses $(9,10)$.

A large variety of newer pulse sequences have been developed to mitigate motion artifacts and other MR imaging 
limitations. Unfortunately, there is no "free lunch" with MRI. For instance, fast spin echo T2 weighted images have less signal to noise, generate less magnetic susceptibility (which may obscure some hematomas) and generate high signal from fat (obscuring some tumors). Combining fat suppression with fast spin echo has helped eliminate the latter. Gradient echo images are prone to excess magnetic susceptibility causing some pathology to appear larger than it actually is while obscuring others. On another MRI technical "battle front" higher field strength magnets have mitigated the never ending struggle to limit signal-to-noise constraints, yet are subject to obscuring from chemical artifact and eddy currents. Newer receiver coil designs continue to improve detection of the MR signal, leading to further improvements in image quality.

Dynamic MR studies acquire sagittal images with the patient in flexion and extension. These may demonstrate atlantoaxial and other intersegment instabilities. In a patient with otherwise unexplained neurologic signs or symptoms, a functional study may reveal cord impingement or nerve root entrapment in only one position.

A systematic approach to post-traumatic imaging must include an understanding of the structures, vulnerable to injury within each phase of the injury mechanics. During the extension phase of whiplash for example, the anterior column will be strained, while the posterior elements are compressed. At the craniocervical junction, the head resists forward acceleration transmitted by the neck. During the flexion phase, the posterior structures (including the PLL, interspinous ligaments, ligamentum nuchae, muscles, and zygapophyseal joint capsule) are subjected to strain while the intervertebral discs and bodies undergo compression (11-14).

The American College of Radiology (ACR) has published Appropriateness Guidelines for imaging in a variety of clinical situations (15). Prima facia, the basic principles of appropriateness guidelines are essential when evaluating cervical spine trauma. An expert multidisciplinary panel reviewed available literature (studies which reported on 15, 000 cervical spine trauma cases) and reached a consensus on imaging choices. This consensus addressed two stages of imaging after acute cervical spine trauma. First, in initial radiographic evaluation is indicated in the acutely symptomatic patient, with or without neurologic deficit. If there is clinical suspicion of ligamentous injury, flexion and extension films are indicated unless there is obvious instability, such as with flexion tear-drop vertebral fracture. Even patients with unstable spinal injuries may not present with immediate neurologic symptoms. Cross-sectional imaging, either MR or CT, is appropriate for patients with neuromuscular changes or radiographic abnormalities suggesting an unstable injury. In particular, for injuries at the craniovertebral junction, the ACR panel recommended CT scanning with multiplanar reconstruction. They were unable to reach a consensus on the use of MR scanning for injuries to the craniovertebral junction.

The ACR criteria mentioned above do not address the patient with delayed onset of symptoms or persistent symptoms after an initial negative screening evaluation. For this group of patients the authors recognize the need to include a third stage of imaging.

General guidelines for imaging do not always address the needs of individual patients. For example, it is well known that claustrophobic patients may become agitated with resultant motion degrading the quality of the MR scan. Pain, hyperactive gag or swallow reflex, erratic respiration, or decreased sensorium may all degrade image quality through patient motion artifact; therefore, it is imperative to use clinical judgment in selecting the appropriate examination for each patient. Imaging of the spine should include the basic principles discussed below.

\section{GENERAL OBSERVATIONS}

When initially evaluating any images, one should inspect the overall quality of the study. Was the area in question adequately surveyed? For example, are there a sufficient number of images, images in multiple planes, osseous and soft tissue window/level settings for CT, and multiple pulse sequences for MRI? Is there poor resolution or degradation of MR images by physiologic or aperiodic motion artifact? If a scan was degraded by patient motion, was an attempt made to repeat the sequences involved, or use alternative sequences less subject to such artifact?

There are a number of important technical factors that affect the quality of MR images. What type scanner was employed and what type of re-and postprocessing factors could affect the quality of the study? If the study was an MRI, was the magnet low-, mid-, or high-field strength? Were appropriate section thicknesses used? What were the field of view, matrix size, and number of excitations? Were appropriate and sufficient pulse sequences obtained in multiple planes? In addition to technical factors, interpersonal factors (patient handling) may greatly influence the quality of an imaging study. Was the patient made comfortable for the exam both physically and psychologically? 


\section{GROSS MORPHOMETRY}

Alterations in alignment may be the "footprints" of trauma and lead to asymmetrical loading and degeneration. Segmentation anomalies should be identified. Major supporting ligaments (such as the anterior longitudinal ligament, annulus, posterior longitudinal ligament), and osseous structures, (including the odontoid, $\mathrm{C} 1$ arch, vertebral bodies, and zygapophyseal joints) are all vulnerable with various loading mechanisms. Hence, it is imperative to exclude changes in alignment, which may be the "telltale" signs of ligamentous or osseous disruption and an unstable spine $(16,17)$.

\section{SOFT TISSUE STRUCTURES}

Myofascial elements should be symmetrical. Muscular asymmetry is present in many common conditions including deformity (psoas asymmetry and scoliosis), infection (psoas abscess), neuromuscular disease and post-operative atrophy. Prevertebral edema or hematoma may be the warning sign of cervical instability, although many cervical spine fractures are not associated with thickened prevertebral tissues. Muscle strains or tears cause characteristic MRI signal changes resulting from edema and/or hemorrhage within the muscle or between the muscles and adjacent structures (12).

Vascular, lymphoid and other prevertebral structures should be identified to exclude associated pathology. Dissection of the vertebral artery may be visible on MR scanning because of the signal hyperintensity of subacute hematoma in a false lumen. MRI is clearly the study of choice in defining a soft tissue tumor (such as sarcoma), although it may be visible on other modalities (albeit radionuclide or CT scan).

\section{OSSEOUS DET AIL}

Fat suppression (i.e., STIR or FSE with fat suppression) MRI pulse sequences can be helpful in evaluating some bone tumors and fractures. MRI is the most sensitive modality for detecting marrow space abnormalities, and consequently might show bone marrow edema as a precursor to a stress fracture. MRI is now recognized as being more sensitive and undoubtedly more specific than radionuclide scanning for discitis or vertebritis. However, plain radiographs are fundamental in furnishing a comprehensive view of anatomic relationships. A single radiograph may lead to an understanding of a three-dimensional structure, which can facilitate the comprehension of cross-sectional images.
An anterior-posterior radiograph of the cervical spine may display the presence of cervical ribs, which clarifies the appearance of an otherwise confusing axial image.

Plain radiographs, sometimes combined with CT scanning, may yield the best appreciation of bone texture and associated features such as periosteal reaction. While MR scanning is more sensitive for detecting bone marrow changes, radiographic and CT findings have higher specificity for identifying bone lesions and in a recent study was found to be more sensitive in detecting cervical spine fracture (14). Normal age related changes in bone marrow most be distinguished from disease and vice versa.

\section{Anterior Column}

The anterior column is the cardinal compression load bearing mechanism. With axial loads to failure, the end-plate will yield, prior to the anulus resulting in intraosseous herniations or compression or burst fractures. Introducing flexion or extension moments (with axial loads) may cause limbus defects or rim fractures. The posterolateral annular margins act as stress risers to torsion. Anular fissures may manifest as a focal area of increased $\mathrm{T} 2$ signal on MRI (i.e., an HIZ) from granulation, neovascularity or inflammation (1). Large radial fissures may allow peripheral migration of nuclear material. While the internal architecture of the disc (nuclear and anular matrix) is optimally appreciated on MRI, CT is equal or superior to MRI in observing the anular contour (as in the setting of disc prolapse). Post discography-CT may provide additional information on the nuclear and anular matrix (18).

An understanding of the whiplash mechanics provides insight into most traumatic and degenerative cervical anterior column pathologies. The anterior column undergoes distraction followed by compression during whiplash. Extension mechanics may lead to an attenuated or avulsed anterior longitudinal ligament, avulsion of the involved vertebrae from the adjacent discs, frank nuclear extrusion, or horizontal rupture of the disc. Annular fissures may result from strain on the annulus. Vertebral body compression, wedge-type ventral compression deformity, and radiographically occult anterior vertebral end-plate fractures result from the flexion phase (19-22).

\section{Posterior Elements}

The posterior elements include the components of the neural arch. Embryologically, the transverse processes are not associated with the neural arch, but for this discussion will 
be included. In the lumbar spine the posterior joint orientation ensures they provide the primary restraining force against axial rotation. Following two to three degrees of rotation they undergo capsular strain, cartilaginous and osseous deformation followed by fracture. Thoracic spine rotation is limited by the constraints of the boney thorax as the joint orientation allows more rotation than the lumbar region. The cervical zygapophyseal joints provide great freedom of motion, allowing the head to rotate and laterally translate. In one study, zygapophyseal joint pain was demonstrated to be the most common cause of chronic neck pain after whiplash injury (23). The zygapophyseal joints share the load with the anterior column in hyperextension injuries. While the anterior column is undergoing distraction, the posterior elements are subject to compression during the extension phase and vice versa for the flexion phase. Compression may lead to cortical defects, fractures, capsular tears, hemorrhage, or rupture of the articular pillars. During the flexion phase, the tips of the spinous processes can be avulsed and interspinous ligaments strained or ruptured.

Posterior element findings may be as gross as "jumped" facets (from fracture/dislocation) or as subtle as facet tropism (signaling chronic torsion stressors or congenital malrotations). Changes in the trabecular architecture or slight distortions of the cortical margins may herald an osteogenic tumor. The osseous aspects of degenerative, erosive or destructive arthropathy such as sclerosis, cortical and joint margin irregularity, capsular calcification, as well as bony remodeling are best imaged by CT, whereas MRI yields greater definition of effusion, synovial cysts and soft tissue processes. Pars defects (spondylolysis) may be missed if the axial scans are interrupted (only through the discs). Hence, the isthmic region should be viewed in multiple planes.

\section{Neuraxial Canals}

Within the spinal column are osteoligamentous conduits, which house neurovascular contents. MRI allows excellent visualization of the intrathecal anatomy, morphology and position of the cord, nerve roots, thecal sac, cord or nerve root edema, hemorrhage, myelomalacia, and syrinx $(21,22)$. MRI also provides superior categorization of Arnold Chiari malformation, MS, neurogenic tumor, epidural hematoma and abscess.

Spinal cord injury manifesting as characteristic signal changes of edema and hemorrhage within the spinal cord on MRI can result from compression of the spinal cord between the ligamentum flavum and retrodisplaced vertebrae above a disrupted disc. Degenerative spondylosis and congenital spinal stenosis may result in spinal cord entrapment, anteriorly by osteophytes or vertebral bodies and posteriorly by thickened or buckled ligamentum flavum. A narrow spinal canal has been shown to correlate with persistent symptoms after whiplash (24).

However, since soft tissue neural impingement (vs. bony encroachment) often leads to the symptoms and signs of spinal stenosis, MRI is a mainstay in observing the soft tissue dimensions of the neuraxial canals. There remains a role of high-resolution, thin section CT in evaluating osseous stenosis, especially in the cervical spine, where the following pitfalls of MRI: variable signal intensity in degenerative osseous ridges, magnetic susceptibility, and thicker slices (resulting in partial volume averaging) must be constant. Whether the choice is CT or MR, acquiring images in multiple planes is essential to distinguish various types of stenosis in the setting of mixed pathologies, [central, developmental or acquired, up-down, front-back or, concentric root canal stenosis (albeit entry, mid or exit zone)].

\section{Craniocervical Junction}

The craniocervical junction is the link between the forces acting on the head and the neck. Moreover, forward acceleration of the trunk must be transmitted to the head through the craniocervical junction. As the neck moves forward, the odontoid process impacts the ventral arch of the atlas, which in turn transmits the forces through the atlanto-occipital joint and longus capitis muscles. Any torsion forces imparted in the axial plane will subject the atlantoaxial joint capsules and alar ligaments, as well as vertebral arch to additional strain (Please refer to the sections entitled "CT" and "MRI").

\section{CONCLUSION}

A systematic approach to selecting and applying the appropriate imaging study combined with a careful clinical history and examination will ensure a more accurate diagnosis and proper treatment. The biomechanics of injury and the pathophysiology of the disease process in question should always be considered.

Understanding the unique properties of each diagnostic modality (as well as the combined benefits of various modalities) will enhance the clinician's diagnostic "yield" and ensure cost effectiveness. 
The limitations of spine modalities must also be recognized, for example, the high rate of false-positive findings on MRI, CT and myelography (25). Neuroanatomic/structural information garnered from these studies does not obviate the need for sound clinical judgment and the integration of other studies (such as provocative image-guided injections or eletrodiagnostics), which provide physiological or functional data.

\section{REFERENCES}

1. Aprill C, Bogduk N. High intensity zones in the disc annulus. A sign of painful disc on magnetic resonance imaging. Br J Radiol 1992; 65:361-369.

2. Reid DC, Henderson R, Shaboe Let al. Etiology and clinical course of missed spine fractures. JTrauma 1997; 27:980-986.

3. Wolbarst B. Physics of Radiology, Prentice - Hall International, Inc., 1997.

4. Curry TS, Dowdey JE, Murry RC. Christensen's Physics of Diagnostic Radiology. Fourth Edition, Lea \& Febiger, Philadelphia, 1990.

5. Anthony Brinton Wolbarst. Physics of Radiology. Appleton \& Lange, 1993.

6. Brink JA. Technical aspects of helical (spiral) CT. Radiol Clin North AM 1995; 33:825-841.

7. Genant H. Technical Considerations. In Newton TH, Potts DG (eds). Computed tomography of the spine and spinal cord, Clavadel Press, San Anselmno, 1983: pp 1-14.

8. Volle E, Assheuer J, Hedde JP et. Radicular avulsion resulting from spinal injury: Assessment of diagnostic modalities. Neuroradiology 1992; 34:235-240.

9. Schild HH. MRI made easy (...well almost): Berlex Laboratories Wayne NJ, 1994.

10. Newhouse JH, Wiener JI. Understanding MRI. Little, Brown and Company Boston/Toronto/London, 1991.

11. Johnson G. Hyperextension soft tissue injuries of the cervical spine: A review. J Accid Emerg Med 1996; 13:3-8.

12. LaBan MM. Whiplash: Its evaluation and treatment. Phys Med Rehabil State Art Rev 1990; 4:293-307.

13. Panjabi MM, Cholewicki J, Nibu K et al. Stimulation of whiplash trauma using whole cervical spine specimens. Spine 1998; 23:17-24.

14. Orrison WW, Benzel EC, Willis BK et at. Magnetic resonance imaging evaluation of acute spine trauma. Emerg Radiol 1995; 2:120-128.

15. American College of Radiology Task Force on Appropriateness Criteria: Appropriateness Criteria for Imaging and treatment Decisions. American College of Radiology, 1996.

16. Dvorak J. Functional roentgen diagnosis of the upper cervical spine. Orthopade 1991;20:121-126.

17. Dvorak H, Jayek J, Vehnder R. CT functional diagnostics of the rotary instability of upper cervical spine. Part II: An evaluation of healthy adults with suspected instability. Spine 1987; 12:726-731.

18. Fortin JD, Sehgal N, Nieves R. Lumbar and Thoracic Discography with CT and MRI correlation. In Leonard, $\mathrm{T}$ (ed) Pain Procedures in Clinical Practice, Second Edition. Hanley and Belfus, Philadelphia, 2000: pp 241-264.

19. Davis SJ, Teresi LM, Bradley WG et al. Cervical spine hyperextension injuries: MR findings. Radiology 1991; 180:245-251.

20. Fortin JD, Weber EC. Imaging of whiplash injuries. In Malanga, G(ed). Cervical flexion-extension/whiplash injuries, Hanley \& Belfus, Philadelphia, 1998.

21. Flanders AE, Schaefer DM, Doan JT et al. Acute cervical spine trauma: Correlation of MR imaging findings with degree of neurologic deficit,Radiology 1990; 177:25-33.

22. el-Koury GY, Kathol MH, Daniel WW. Imaging of acute injuries of the cervical spine: Value of plain radiography, CT and MR imaging. Am JRoentgenol 1995; 164:43-50.

23. Barnsley L, Lord SM, Wallis BJ et al. The prevalence of chronic zygapophyseal joint pain after whiplash. Spine 1995; 20:20-26.

24. Pettersson K, Karrholm J, Toolanen G et al. Decreased width of the spinal canal in patients with chronic symptoms after whiplash. Spine 1995; 20:1664-1667.

25. Boden SD, Davis DO, Dina TS et al. Abnormal magnetic resonance scans of the lumbar spine in asymptomatic subjects. A prospective investigation. JBone Joint Surg 1990; 72:403-408. 